\title{
Recent advances in Immunobullous diseases
}

Bhari $N^{1}$, Verma $K K^{2}$

${ }^{1}$ Senior Resident $\&{ }^{2}$ Professor, Department of Dermatology and Venereology, All India Institute of Medical Sciences, India.

\begin{abstract}
The researches in the recent past have led to better understanding of pathogenesis, diagnostic methods and treatment of autoimmune bullous diseases. Significant advances in our understanding of pathogenesis of pemphigus have resulted in development of multiple pathogenic models and discovery of new signalling pathways involved in its pathogenesis. Various newer clinical variants have been described and diagnostic methods have been developed. Disease activity scoring methods in pemphigus provide comparable and interpretable results which facilitate better understanding of disease activity and assessment of treatment response. Use of biological agents have led to better control of disease activity and other newer therapeutic options are being explored.
\end{abstract}

Keywords: Immunobullous diseases, Recent advances, Pathogenesis, Diagnosis, Scoring, Treatment

\section{Address for correspondence}

Prof. Kaushal Verma, M.D.

Department of Dermatology and Venereology

All India Institute of Medical Sciences,

New Delhi, 110029, India.

Email: prokverma@hotmail.com

\section{Introduction}

Immunobullous diseases are severe blistering conditions affecting the skin and often mucous membranes. These can be intraepidermal or subepidermal, depending on the level of split in the skin. The researches in the recent past have led to better understanding of pathogenesis, diagnostic methods and treatment of autoimmune bullous diseases. This review will focus on developments in the pathogenesis, newer clinical variants, severity scoring, diagnostic techniques and advances in the management of these diseases.

\section{Pathogenesis}

The desmosome is an intercellular junction which mediates strong adhesion and anchors the intermediate filament cytoskeleton to the plasma membrane at sites of cell to cell contact. Adhesive interactions in the desmosomes are mediated by desmogleins and desmocollins, which are the members of the cadherin superfamily of adhesion molecules. Desmosomal plaque proteins, including plakoglobin and desmoplakin, tether the cytoplasmic tails of the desmoglein and desmocollin to the intermediate filament cytoskeleton. This architectural arrangement is essential for maintaining cell integrity and its interaction with the adjacent cells. ${ }^{1}$

\section{Pathogenesis of Intra-epidermal bullous diseases}

Intra-epidermal bullous diseases are caused due to loss of cell adhesion leading to acantholysis.

\section{Conventional theories for acnatholysis}

Conventionally stearic hindrance and plasminogen activation were considered most important factors responsible for acantholysis. 


\section{Review Article}

- $\quad$ Stearic hindrance: Binding of IgG autoantibodies to keratinocytes blocks the amino-terminal domain of desmogleins causing direct inhibition of desmosomal cadherin, leading to acantholysis.

- Plasminogen activator: The antigenantibody complex induces production of plasminogen activator, which in turn leads to the production of active plasmin. The active plasmin causes cytoskeletal damage and cell dissociation.

\section{Newer concepts in acantholysis}

Now multiple signaling pathways involving p38 mitogen activated protein kinase (MAPK), RhoA, protein kinase $\mathrm{C}$ (PKC), c-myc, and plakoglobin have been shown to play a role in the pathogenesis of pemphigus.

1. P38 signaling mediated acantholysis P38 MAPK signaling and Dsg3 internalization are linked events causing acantholysis in pemphigus. Binding of antidesmoglein antibodies to desmosome activates p38 MAPK signaling pathway, resulting in phosphorylation of heat shock protein 27 (hsp27) which leads to reorganization of actin and keratin causing acantholysis. $^{2}$ Cells treated with the p38 MAPK inhibitor when exposed to pemphigus auto antibodies, did not have clustering or loss of cell surface Dsg3. Therefore p38 MAPK inhibitors may be potential therapeutic options in the management of pemphigus. ${ }^{3}$

\section{Role of epidermal growth factor receptor (EGFR)}

Epidermal growth factor receptor is a mediator of p38 MAPK pathway and is activated by binding of pemphigus vulgaris auto-antibodies to the keratinocytes. Inhibition of EGFR blocks antibody mediated acantholysis in pemphigus. Since the use of p38 inhibitors in clinical trials got limited due to hepatotoxicity of these compounds, EFGR inhibitors can still be used to inhibit this pathway of acantholysis. ${ }^{4}$
EGFR inhibitors like erlotinib and lapatinib have been shown to decrease epidermal blistering activity in experimental models. Lapatinib is a dual EGFR and ErbB2 inhibitor. The dual-nature of lapatinib inhibition results in more effective control of skin as well as mucosal blistering. However, the beneficial effect is observed only when EGFR in the epidermis is inhibited in the range of $36-69 \%$ of its normal activity (median 52\%) while this effect is lost at higher inhibition following a non-linear, V-shaped dose-response curve. ${ }^{5}$

3. Desmoplakin point mutation enhancing keratinocyte adhesion

Desmosomal plaque proteins plakoglobin and desmoplakin, tether the cytoplasmic tails of the desmosomal cadherins to the intermediate filament cytoskeleton. A specific desmoplakin (DP) mutation S2849G, in which serine residue is replaced by glycine, results in increased anchorage of keratin filaments. This mutated desmoplakin becomes less prone to protein kinase $\mathrm{C}$ induced phosphorylation, which occurs when pemphigus auto-antibodies bind to keratinocytes. Thus keratinocytes expressing DP-S2849G-GFP were found to be less susceptible to pathogenic effects of pemphigus autoantibodies. Therefore inhibition of protein kinase $\mathrm{C}$ induced phosphorylation of desmoplakin may be of therapeutic value. ${ }^{6}$

4. Antibodies against keratinocyte acetylcholine receptors (AchR) - AchR $\alpha 9$ and Pemphaxin (PX)

Recently it has been shown that desmogleins are not the lone target for pemphigus autoantibodies. Various other target molecules have now been identified, which are responsible for blistering in pemphigus. Antibodies against keratinocyte acetylcholine receptors (AchR) - AchR $\alpha 9$ and pemphaxin (PX) have been discovered in about $85 \%$ patients with pemphigus. 


\section{Review Article}

Pemphigus auto-antibodies binding to $\mathrm{Ach} R$ $\alpha 9$ blocks $\mathrm{Ca}^{++}$influx involved in desmosome assembly leading to desmosomal dissociation. It also results in protein kinase $\mathrm{C}$ (PKC) activation, which causes phosphorylation of structural and adhesion molecules resulting in cytoskeletal collapse and weakening of intercellular junctions respectively. AchR agonist have been shown to reduce blistering in pemphigus, which further confirms their role in pathogenesis of pemphigus. ${ }^{7}$

\section{Role of $B$ reg cells}

In pemphigus, $\mathrm{CD}^{+} \mathrm{T}$ cells stimulate $\mathrm{B}$ cells for pathogenic $\mathrm{IgG}$ production. $\mathrm{CD} 19^{+}$ $\mathrm{CD} 24^{+} \mathrm{CD} 38^{+} \mathrm{B}$ reg cells levels are significantly higher but the capability of IL-10 production is significantly impaired in these patients compared to healthy controls. Also, these B reg cells are functionally impaired to down regulate IFN- $\alpha$ production by $\mathrm{CD}^{+}{ }^{+} \mathrm{T}$ cells. Thus $B$ regs cells can be an important therapeutic target in the management of pemphigus. ${ }^{8}$

\section{Role of the cellular immune system}

Cellular immunity has also been shown to provide protection against development of pemphigus. CD28 is a co-stimulatory signal for T-cell activation and CD28- deficient mice are found to be 5 fold more sensitive to develop pemphigus than wild-type mice. ${ }^{9}$ CD 200, which play an important role in maintenance of pro-inflammatory reaction and immune tolerance in the body, is also implicated in pathogenesis of pemphigus, therefore CD200 may also be a potentially important therapeutic target. ${ }^{10}$

\section{Theories on the pathogenesis of pemphigus}

\section{Multiple hits hypothesis}

Recent studies have demonstrated that multiple type of antibodies are involved in the pathogenesis of pemphigus such as antibodies to desmosomal proteins like desmogleins, desmocollins, plakins and nondesmosomal proteins as antimitochondrial antibodies, antibodies to cell-membrane receptor, nicotinic acetylcholine receptor, pemphaxin, thyroperoxidase and some other annexins.

According to this theory, pemphigus is a complex disease, initiated by at least three classes of auto-antibodies directed against desmosomal, mitochondrial and other keratinocyte autoantigens resulting in acantholysis. $^{11}$

\section{The antibody-induced apoptosis theory} The mechanisms of apoptosis in pemphigus may be based on the auto-antibody triggered activation of signaling pathways, such as FasR pathway, leading to an increase in levels of intracellular FasR, FasL, Bax and p53 and decrease in the levels of Bcl-2; enrichment of caspase-8; and activation of caspases 1 and 3 . Inhibitors of caspases 1 or 3 inhibit IgG-mediated apoptosis and blocks acantholysis thereby supporting the role of apoptosis in pathogenesis of pemphigus. ${ }^{11}$

\section{The basal-cell shrinkage hypothesis and the apoptolysis theory}

According to this theory, after the pathogenic pemphigus auto-antibodies bind to the keratinocyte receptors, a series of signal-transduction pathways trigger the rupture of the cytoskeleton, which results in the collapse and shrinkage of the keratinocytes, known as "apoptolysis". ${ }^{11}$

\section{Pathogenesis of sub-epidermal bullous diseases}

\section{IgE antibodies in bullous pemphigoid}

Bullous pemphigoid (BP) is an autoimmune subepidermal blistering disease characterized by IgG auto-antibodies against the hemidesmosomal proteins BP180 (type XVII collagen) and BP230. These antibodies result in complement activation and neutrophil recruitment with subsequent release of elastase and gelatinase $\mathrm{B}$ at the lesional site. However, BP is also found to be associated with Th2-type of immune responses and production of $\operatorname{IgE}$ autoantibodies. These antibodies trigger mast 


\section{Review Article}

cells and basophils to release proinflammatory mediators, such as histamine which may be responsible for itching, erythema and urticarial lesions in these patients. $^{12}$

\section{Up-regulation of Hsp90 in bullous pemphigoid}

Bullous pemphigoid auto-antibodies induces production of inflammatory cytokine such as interleukin- 6 and interleukin- 8 in the keratinocytes. Release of IL-6 and IL-8 leads to chemotaxis-induced granulocyte recruitment, essential for blister formation. This inflammatory pathway is mediated by up-regulation Hsp90 molecule in these cells. Pharmacological blockade of Hsp90 could therefore represent a promising therapeutic approach, interfering with this key inflammatory cascade in $\mathrm{BP} .{ }^{13}$

\section{Newly described pemphigus clinical types of autoimmune bullous diseases}

\section{Dyshidrosiform vulgaris}

This variant is characterized by vesicobullous lesions predominantly on the palms and soles. Histopathology and immunofluorescence shows characteristic features of pemphigus vulgaris. ${ }^{14}$

\section{Paraneoplastic autoimmune multiorgan syndrome (PAMS)}

In 2001, a new term paraneoplastic autoimmune multi-organ syndrome (PAMS) was coined for paraneoplastic pemphigus disease, to reflect the presence of target antigens and the pathological damage which frequently occurs in multiple organ systems like lungs, kidneys and muscles in this disease. ${ }^{15}$

Diagnostic criteria for PAMS were revised as: Major criteria

1. Polymorphous mucocutaneous eruption

2. Concurrent internal neoplasia

3. Characteristic serum immunoprecipitation findings

Minor criteria

1. Positive cytoplasmic staining of rat bladder epithelium by indirect immunofluorescence

2. Intercellular and basement membrane zone immunoreactants on direct immunofluorescence of perilesional tissue

3. Acantholysis in biopsy specimen from at least one anatomic site of involvement *Diagnosis of neoplasia-induced pemphigus is made when three major or two major and two minor criteria are met.

\section{IgA Paraneoplastic pemphigus}

Paraneoplastic pemphigus is usually associated with IgG1 antibodies against desmoplakin 1 and 2, envoplakin, periplakin, bullous pemphigoid antigen 1 , pectin, desmoglein 1 and 3. Recently, IgA antibodies reactive with $\mathrm{BP} 230,210-\mathrm{kDa}$ envoplakin and periplakin were also seen in addition to $\operatorname{IgG}$ antibodies in a case of paraneoplastic pemphigus associated with angio-immunoblastic T cell lymphoma. ${ }^{16}$ This condition was described as $\operatorname{IgA}$ paraneoplastic pemphigus.

\section{Anti-laminin gamma-1 pemphigoid} Anti-laminin gamma-1 pemphigoid is an autoimmune subepidermal bullous disease which was previously known as anti-p200 pemphigoid. It is characterized by circulating and tissue-bound auto-antibodies to a 200-kDa protein (p200) of the dermal-epidermal junction (DEJ). The identity of p200 has now been unmasked as laminin $\gamma-1$, so this disease has been renamed as anti-laminin gamma-1 pemphigoid.

Clinical presentation of anti-laminin gamma-1 pemphigoid closely resembles bullous pemphigoid. Histopathology shows subepidermal blistering and superficial inflammatory infiltrate of predominantly neutrophils. Direct immunofluorescence of perilesional skin demonstrates linear deposits of IgG and C3 along the DEJ. $\mathrm{NaCl}$-split skin shows anti-laminin gamma1 pemphigoid immunoglobulin on the dermal side of basement membrane on 


\section{Review Article}

indirect immunofluorescence microscopy. A $200-\mathrm{kDa}$ protein (p200) of the normal human dermis is demonstrated in sera of all patients with anti-p200 pemphigoid on immunoblotting. ${ }^{17}$

\section{Anti-epilligrin pemphigoid}

Anti-epilligrin pemphigoid (AECP) is a chronic, autoimmune sub-epithelial blistering disease, primarily involving mucous membrane. It comprises of 5-20\% cases of mucous membrane pemphigoid. It is characterized by circulating antibasement membrane auto-antibodies to laminin 5. Identification of this variant is important due to its association with malignancy in $30-40 \%$ of the cases. The malignancy is usually diagnosed within one year of onset of blisters. Adenocarcinoma of the lung, stomach, colon, uterus are most commonly associated malignancies. ${ }^{18}$

Several new clinical variants of bullous pemphigoid have also been described like dyshidrosiform pemphigoid with isolated palmoplantar involvement, dermatitis herpetiformislike bullous pemphigoid (vesicular pemphigoid), erythrodermic bullous pemphigoid, lichen planus pemphigoides, gestational pemphigoid and pemphigoid incipiens (seen in elderly patients with itchy BP like lesions but a negative DIF in the presence of circulating anti-BP180 and/or BP230 antibodies).

\section{New scoring systems in immunobullous disorders}

Pemphigus Disease Activity Measurements: Pemphigus Disease Area Index (PDAI), Autoimmune Bullous Skin Disorder Intensity Score (ABSIS), and Pemphigus Vulgaris Activity Score (PVAS)

Disease activity indexes are important in clinical studies to provide comparable, interpretable results and to facilitate evidence-based decision making for physicians. Pemphigus Disease Area Index (PDAI) assigns scores to defined anatomical regions based on the number and size of the lesions. The PDAI score has a potential range of 0 to 263 (120 points for skin activity, 10 points for scalp activity, 120 points for mucosal activity, and 13 points for post-inflammatory hyperpigmentation $[\mathrm{PIH}]$, representing disease damage). ${ }^{19}$

Autoimmune Bullous Skin Disorder Intensity Score (ABSIS) takes into account body surface area and the type of lesions as well as discomfort during eating and drinking. The ABSIS has a potential range of 0 to 206 (150 points for skin involvement, 11 points for oral involvement, and 45 points for subjective oral discomfort). ${ }^{20}$ Pemphigus Vulgaris Activity Score (PVAS) measures the number of lesions, the types of lesions and the involvement of defined anatomical regions. The Nikolsky's sign is also incorporated in skin activity scoring. The PVAS has a potential range of 0 to 18 (11 points for skin activity and 7 points for mucosal activity). ${ }^{21}$

In a comparative study of these three scoring systems, PDAI was found to have highest validity and is recommended for use in the multicenter studies for rare diseases, such as pemphigus vulgaris. ${ }^{22}$

\section{Treatment of Autoimmune Bullous Disease} Quality of Life questionnaire (TABQOL) It is a valid and reliable instrument which may be used to measure the treatment burden in Autoimmune Bullous Disease (AIBD). It contains 17 items which can be used to quantify the impact of a patient's treatment on his quality of life (QOL). ${ }^{23}$

Diagnosis of autoimmune bullous diseases Pemphigus vulgaris \& Pemphigus foliaceus 1. Direct immunofluorescence (DIF) on outer root sheath of hair follicle Pemphigus antigens are distributed throughout the outer root sheath (ORS) and in the dermal bulb matrix cells of hair follicle. Dsg1 is expressed in epidermal suprabasal cells, inner root sheath and the innermost layers of the ORS whereas Dsg3 is expressed throughout the ORS.

DIF pattern of the ORS of hair samples is 


\section{Review Article}

similar to the skin in pemphigus. Though both anagen and telogen hair give high yield, telogen hair are preferred as it requires gentle pulling rather than plucking of anagen hair. It is a feasible, cost-effective and noninvasive alternative to the perilesional skin for DIF which can be used in almost all variants of pemphigus. ${ }^{24}$

\section{Desmoglein 1,3 enzyme linked immunosorbent assay (ELISA)}

Anti-Dsg1 and anti-Dsg3 ELISA is shown to be superior to DIF in detecting immunological remission and its correlation with severity of the disease in pemphigus. $^{25,26}$

\section{Immunoblotting}

Immunoblotting (IB) is helpful in the diagnosis of autoimmune bullous disease by enabling recognition of a wide range of skin antigens based on molecular weight separation on the panel. The human cell line A431, a differentiated adult epidermoid cell line, is used for this procedure. It has been found to have high sensitivity in the diagnosis of AIBD. ${ }^{27}$

\section{Scanning electron microscopy}

Roof of blister from pemphigus shows loss of adhesion between keratinocytes as well as acantholytic keratinocytes with polygonal or round borders, under scanning electron microscope. These changes are not seen in a blister roof of subepidermal blistering disease. $^{28}$

\section{Reflectance confocal microscopy}

Reflectance confocal microscopy (RCM) is a novel, non-invasive imaging technique. Imaging of the epidermis, appendages as well as the upper dermis can be performed using RCM.

RCM images show abnormal cornified layer, disarranged granular and spinous layers and clefts (bullae) in the suprabasilar layers which contains bright, round to oval acatholytic keratinocytes in pemphigus vulgaris. Also there are Inflammatory cells in the epidermis and superficial dermis with dilated blood vessels in the upper dermis. Detachment of the outer root sheath in hair follicles is seen on RCM in pemphigus vulgaris, whereas in pemphigus foliaceus, the cleft is localized to stratum granulosum. ${ }^{29}$

6. Ultrastructural immunocytochemistry/ Immuno-electron microscopy

In this technique antibodies recognise specific antigens of the dermo-epidermal junction. Immune deposits are seen on the desmosomal plaques in pemphigus vulgaris, whereas in bullous pemphigoid and pemphigoid gestationis deposits are seen in lamina lucida, In epidermolysis bullous acquisita these deposits are seen in lamina densea, however cicatricial pemphigoid and linear IgA disease show immune deposits in both lamina lucida and lamina densa. ${ }^{30}$

\section{Bullous Pemphigoid}

\section{Paper-Based ELISA}

It is a rapid, low-cost, widely applicable ELISA-based system to detect the NC16A antibody using filter paper and wax printer. The test can be performed on serum as well as blister fluid and requires only $2 \mu \mathrm{L}$ of fluid. It is a rapid test which take 70 minutes for the procedure, compared to 150 minutes of conventional ELISA with comparable sensitivity and specificity. It can also be used to monitor treatment response in bullous pemphigoid. ${ }^{31}$

\section{DNA microarray scanner}

DNA microarray scanner can be used for reading the immunofluorescence findings in bullous diseases. It produces diagnostic images of immune deposits and the results show concordance with conventional immunofluorescence microscopy. ${ }^{32}$

\section{Treatment of autoimmune bullous diseases 1. Rituximab \\ Rituximab is a chimeric monoclonal}


Review Article

antibody which targets the CD20 molecule on B cells. Two most common dosing protocols described for rituximab are lymphoma and rheumatoid arthritis (RA) protocol. The lymphoma protocol consists of $375 \mathrm{mg} / \mathrm{m}^{2}$ of rituximab per week for 4 weeks. The RA protocol consists of two doses of 1,000 mg of drug at two weeks apart. Both protocols have shown their efficacy in pemphigus vulgaris and pemphigus foliaceus. ${ }^{33}$ Mixed protocol has also been used in some studies. The infusions are given after baseline investigations, under monitoring.

There are few case reports of use of rituximab in mucous membrane pemphigoid. Rituximab with IVIG has been reported to be effective in ocular cicatricial pemphigoid. ${ }^{34}$ Though it has been infrequently used in bullous pemphigoid. The potential indications of rituximab in BP may be: (1) recalcitrant disease and (2) patients with multiple relapses. ${ }^{35}$

\section{Intravenous immunoglobulin}

Intravenous immunoglobulin (IVIG) act through various mechanisms like modulation of expression and function of Fc receptors, interference with complement activation, modulation of dendritic cell, $\mathrm{T}$ and $\mathrm{B}$ cell activation, differentiation and their effector functions. It exerts immunemodulatory effects without suppressing the immune system, which provides a distinctive advantage over conventional treatment. The dose of IVIG is $2 \mathrm{gm} / \mathrm{kg}$ over 5 consecutive days.

Multiple case series and a single randomised controlled trial has shown efficacy of IVIG in pemphigus vulgaris. ${ }^{36}$ It has also been shown to be effective in bullous pemphigoid, mucous membrane pemphigoid and epidermolysis bullous acquisita. $^{37}$

\section{Immunoadsorption}

Immunoadsorption (IA) consists of collecting patient's plasma, passing it through an absorbing column (immunoadsorption using a regenerable staphylococcal protein A) to remove circulating immune complexes and antibodies and then returning the filtered plasma to the patient. One IA cycle of three consecutive days is followed by additional cycles after 3 weeks and then at 4 -week intervals until lesions heal by $90 \%$. Immunoadsorption with tryptophan-linked poly-vinyl alcohol absorbers are used nowadays. A single immunoadsorption treatment has shown to reduce antidesmoglein auto-antibodies levels by about $30 \%$. It efficacy has been shown in the treatment of pemphigus vulgaris, pemphigus foliaceous, IgA pemphigus and bullous pemphigoid. ${ }^{38}$

\section{TNF-alpha antagonists}

TNF-alpha plays a role in the acantholytic process, therefore TNF-alpha antagonists may be beneficial in the treatment of pemphigus. Infliximab in a dose of $5 \mathrm{mg} / \mathrm{kg}$ in weeks 0,2 , and 6 , and then every 8 weeks have been successfully used in pemphigus. Etanercept was tested in a 16-week randomized, double-blind, placebocontrolled phase II study as a steroid-sparing drug. ${ }^{39}$ Adalimumab has also shown its efficacy in pemphigus, in a few case reports. ${ }^{40}$

\section{Cholinergic agonists}

Cholinergic agonist like carbachol $(40 \mu \mathrm{g} / \mathrm{kg}$ body weight) and pyridostigmine bromide (360 mg/day) have shown upregulation of adhesion molecules and reduce severity of blistering in pemphigus. ${ }^{41}$

\section{Alemtuzumab}

Alemtuzumab is a monoclonal antibody which inhibits CD52. It has shown to cause good improvement in case of paraneoplastic pemphigus associated with chronic 


\section{Review Article}

lymphocytic leukemia. Alemtuzumab infusion is started at a low dose of $3 \mathrm{mg}$ daily intravenously and if this infusion is tolerated well without any infusion reaction, the dose is slowly increased over a period of one week to $30 \mathrm{mg}$, which is given subcutaneously three times weekly for 12 weeks. Lymphopenia and secondary infections are important known complications of this agent. ${ }^{42}$

\section{Mizoribine}

It is a novel purine synthesis inhibitor and has been found to be effective as an adjuvant for the treatment of pemphigus vulgaris. There is substantial reduction in the dose and side effects of the corticosteroid therapy with mizoribine. ${ }^{43}$

\section{Conclusion}

New researches have led to discovery of multiple new signalling pathways involved in pathogenesis of pemphigus and various signalling molecules such as p38 mitogen activated protein kinase (MAPK), epidermal growth factor receptor, acetylcholine receptors - AchR $\alpha 9$ and pemphaxin (PX) have been shown to mediate acantholysis. Cellular immunity has also been found to play an important role in pathogenesis of autoimmune bullous diseases. New clinical variants such as dyshidrosiform vulgaris, paraneoplastic autoimmune multiorgan syndrome, IgA paraneoplastic pemphigus, anti-laminin gamma1 pemphigoid, anti-epilligrin pemphigoid, dyshidrosiform pemphigoid, dermatitis herpetiformis-like bullous pemphigoid, erythrodermic bullous pemphigoid, lichen planus pemphigoides, gestational pemphigoid and pemphigoid incipient have been described as well as new diagnostic techniques such as direct immunofluorescence on outer root sheath of hair follicle, reflectance confocal microscopy, immunoelectron microscopy, paper-Based ELISA and DNA microarray scanner etc. have been developed. Use of biological agents such as rituximab, TNFalpha antagonists and alemtuzumab have led to better control of disease activity and newer therapeutic options like immunoadsorption, cholinergic agonists and mizoribine etc. are being explored.

\section{References}

1. Al-Jassar C, Bikker H, Overduin M, Chidgey M. Mechanistic basis of desmosome-targeted diseases. J Mol Biol 2013; 425: 4006-22. http://dx.doi.org/10.1016/j.jmb.2013.07.035

2. Stokoe D, Engel K, Campbell DG, Cohen P, Gaestel M. Identification of MAPKAP kinase 2 as a major enzyme responsible for the phosphorylation of the small mammalian heat shock proteins. FEBS Lett 1992; 313: 307-13. http://dx.doi.org/10.1016/0014-5793(92)81216-9

3. Mao X, Li H, Sano Y, Gaestel M, Mo Park J, Payne AS. MAPKAP kinase 2 (MK2)-dependent and independent models of blister formation in pemphigus vulgaris. J Invest Dermatol 2014; 134: 68-76. http://dx.doi.org/10.1038/jid.2013.224

4. Bektas M, Jolly PS, Berkowitz P, Amagai M, Rubenstein DS. A pathophysiologic role for epidermal growth factor receptor in pemphigus acantholysis. J Biol Chem 2013; 288: 9447-56. http://dx.doi.org/10.1074/jbc.M112.438010

5. Sayar BS, Rüegg S, Schmidt E, Sibilia M, Siffert M, Suter MM, et al. EGFR inhibitors erlotinib and lapatinib ameliorate epidermal blistering in pemphigus vulgaris in a non-linear, V-shaped relationship. Exp Dermatol 2014; 23: 33-8. http://dx.doi.org/10.1111/exd.12290

6. Dehner C, Rötzer V, Waschke J, Spindler V. A desmoplakin point mutation with enhanced keratin association ameliorates pemphigus vulgaris autoantibody-mediated loss of cell cohesion. Am J Pathol 2014; 184: 2528-36. http://dx.doi.org/10.1016/j.ajpath.2014.05.016

7. Nguyen VT, Ndoye A, Grando SA. Novel human alpha9 acetylcholine receptor regulating 


\section{Review Article}

keratinocyte adhesion is targeted by Pemphigus vulgaris autoimmunity. Am J Pathol 2000; 157: 1377-91. http://dx.doi.org/10.1016/S0002-9440(10)64651-2

8. Zhu HQ, Xu RC, Chen YY, Yuan HJ, Cao H, Zhao XQ, et al. Impaired Function of CD19+ CD24hi CD38hi Regulatory B Cells in Pemphigus Patients. Br J Dermatol 2015; 172: 101-10. http://dx.doi.org/10.1111/bjd.13192

9. Toto P, Feliciani C, Amerio P, Suzuki H, Wang B, Shivji GM, et al. Immune modulation in pemphigus vulgaris: role of CD28 and IL-10. J Immunol 2000; 164: 522-9. http://dx.doi.org/10.4049/jimmunol.164.1.522

10. Akman-Karaka ${ }^{\circ}$ A, Yalcin AD, Koç S, Gumuslu S, Senol YY, Özkesici B, et al. There might be a role for CD200 in the pathogenesis of autoimmune and inflammatory skin disorders. Med Sci Monit 2013; 19: 888-91. http://dx.doi.org/10.12659/MSM.889624

11. Pan M, Liu X, Zheng J. The pathogenic role of autoantibodies in pemphigus vulgaris. Clin Exp Dermatol 2011; 36: 703-7. http://dx.doi.org/10.1111/j.1365-2230.2011.04092.x

12. Dresow SK, Sitaru C, Recke A, Oostingh GJ, Zillikens D, Gibbs BF. IgE autoantibodies against the intracellular domain of BP180. Br J Dermatol 2009; 160: 429-32. http://dx.doi.org/10.1111/j.1365-2133.2008.08858.x

13. Tukaj S, Grüner D, Zillikens D, Kasperkiewicz M. Hsp90 blockade modulates bullous pemphigoid IgG-induced IL-8 production by keratinocytes. Cell Stress Chaperones 2014; 19: 887-94. http://dx.doi.org/10.1007/s12192-014-0513-8

14. Gharami RC, Kumar P, Mondal A, Ghosh K. Dyshidrosiform Pemphigus vulgaris: Report of an unusual case. Dermatol Online J 2010; 16: 10.

15. Billet SE, Grando SA, Pittelkow MR. Paraneoplastic autoimmune multiorgan syndrome: Review of the literature and support for a cytotoxic role in pathogenesis. Autoimmunity 2006; 39: 61730. http://dx.doi.org/10.1080/08916930600972099

16. Yashiro M, Nakano T, Taniguchi T, Katsuoka K, Tadera N, Miyazaki K, et al. IgA paraneoplastic pemphigus in angioimmunoblastic T-cell lymphoma with antibodies to desmocollin 1, type VII collagen and laminin 332. Acta Derm Venereol 2014; 94: 235-6. http://dx.doi.org/10.2340/00015555-1660

17. Dainichi T, Koga H, Tsuji T, Ishii N, Ohyama B, Ueda A, et al. From anti-p200 pemphigoid to anti-laminin gammal pemphigoid. J Dermatol 2010; 37: 231-8. http://dx.doi.org/10.1111/j.13468138.2009.00793.x

18. Fukushima S, Egawa K, Nishi H, Wakasugi S, Ishii N, Hashimoto T, et al. Two cases of antiepiligrin cicatricial pemphigoid with and without associated malignancy. Acta Derm Venereol 2008; 88: 484-7. http://dx.doi.org/10.2340/00015555-0506

19. Rosenbach M, Murrell DF, Bystryn JC, Dulay S, Dick S, Fakharzadeh S, et al. Reliability and convergent validity of two outcome instruments for pemphigus. J Invest Dermatol 2009; 129: 2404-10. http://dx.doi.org/10.1038/jid.2009.72

20. Pfütze M, Niedermeier A, Hertl M, Eming R. Introducing a novel Autoimmune Bullous Skin Disorder Intensity Score (ABSIS) in pemphigus. Eur J Dermatol 2007; 17: 4-11.

21. Agarwal M, Walia R, Kochhar AM, Chander R. Pemphigus Area and Activity Score (PAAS): A novel clinical scoring method for monitoring of pemphigus vulgaris patients. Int J Dermatol 1998; 37: 158-60. 


\section{Review Article}

22. Rahbar Z, Daneshpazhooh M, Mirshams-Shahshahani M, Esmaili N, Heidari K, Aghazadeh N, et al. Pemphigus disease activity measurements: pemphigus disease area index, autoimmune bullous skin disorder intensity score, and pemphigus vulgaris activity score. JAMA Dermatol 2014; 150: 266-72. http://dx.doi.org/10.1001/jamadermatol.2013.8175

23. Tjokrowidjaja A, Daniel BS, Frew JW, Sebaratnam DF, Hanna AM, Chee S, et al. The development and validation of the treatment of autoimmune bullous disease quality of life questionnaire, a tool to measure the quality of life impacts of treatments used in patients with autoimmune blistering disease. Br J Dermatol 2013; 169: 1000-6. http://dx.doi.org/10.1111/bjd.12623

24. Kumaresan M, Rai R, Sandhya V. Immunofluorescence of the outer root sheath: an aid to diagnosis in pemphigus. Clin Exp Dermatol 2011; 36: 298-301. http://dx.doi.org/10.1111/j.13652230.2010.03981.x

25. Daneshpazhooh M, Kamyab K, Kalantari MS, Balighi K, Naraghi ZS, Shamohammadi S, et al. Comparison of desmoglein 1 and 3 enzyme-linked immunosorbent assay and direct immunofluorescence for evaluation of immunological remission in pemphigus vulgaris. Clin Exp Dermatol 2014; 39: 41-7. http://dx.doi.org/10.1111/ced.12187

26. Mortazavi H, Shahdi M, Amirzargar AA, Naraghi ZS, Valikhani M, Daneshpazhooh M, et al. Desmoglein ELISA in the diagnosis of pemphigus and its correlation with the severity of pemphigus vulgaris. Iran J Allergy Asthma Immunol 2009; 8: 53-6.

27. Khandpur S, Sharma VK, Sharma A, Pathria G, Satyam A. Comparison of enzyme-linked immunosorbent assay test with immunoblot assay in the diagnosis of pemphigus in Indian patients. Indian J Dermatol Venereol Leprol 2010; 76: 27-32. http://dx.doi.org/10.4103/03786323.58675

28. Almeida Jr HL, Leitão AH, Rossi G, Rocha NM, Marques e Silva R. Scanning electron microscopy of acantholysis in pemphigus foliaceus. An Bras Dermatol 2013; 88: 456-8. http://dx.doi.org/10.1590/abd1806-4841.20132047

29. Alessi SS, Nico MM, Fernandes JD, Lourenço SV. Reflectance confocal microscopy as a new tool in the in vivo evaluation of desquamative gingivitis: patterns in mucous membrane pemphigoid, pemphigus vulgaris and oral lichen planus. Br J Dermatol 2013; 168: 257-64. http://dx.doi.org/10.1111/bjd.12021

30. Karpouzis A, Vamvassakis E, Stavrianeas N, Koumantaki-Mathioudaki E, Karpouzi M, Vareltzides A. Ultrastructural immunocytochemistry of autoimmune bullous diseases. Australas J Dermatol. 2002; 43: 113-9. http://dx.doi.org/10.1046/j.1440-0960.2002.00571.x

31. Hsu CK, Huang HY, Chen WR, Nishie W, Ujiie H, Natsuga K, et al. Paper-based ELISA for the detection of autoimmune antibodies in body fluid-the case of bullous pemphigoid. Anal Chem 2014; 86: 4605-10. http://dx.doi.org/10.1021/ac500835k

32. Iwamoto S, Iwamoto AT, Cha J, Clark T, Lu M, Breen C, et al. The utility of the DNA microarray scanner to simplify the immunofluorescence evaluation of autoimmune bullous diseases. Am J Dermatopathol 2009; 31: 218-22. http://dx.doi.org/10.1097/DAD.0b013e31818ffde1

33. Zakka LR, Shetty SS, Ahmed AR. Rituximab in the Treatment of Pemphigus Vulgaris. Dermatol There 2012; 2: 17. http://dx.doi.org/10.1007/s13555-012-0017-3

34. Heelan K, Walsh S, Shear NH. Treatment of mucous membrane pemphigoid with rituximab. J Am Acad Dermatol 2013; 69: 310-1. http://dx.doi.org/10.1016/j.jaad.2013.01.046 


\section{Review Article}

35. Wang TS, Tsai TF. Remission of bullous pemphigoid after rituximab treatment in a psoriasis patient on regular low-dose methotrexate. Acta Derm Venereol 2014; 94: 108-9. http://dx.doi.org/10.2340/00015555-1619

36. Amagai M, Ikeda S, Shimizu H, lizuka H, Hanada K, Aiba S, et al. A randomized double-blind trial of intravenous immunoglobulin for pemphigus. J Am Acad Dermatol 2009; 60: 595-603. http://dx.doi.org/10.1016/j.jaad.2008.09.052

37. Tuchinda P, Ritchie S, Gaspari AA. Bullous pemphigoid treated with intravenous immunoglobulin. Cutis 2014; 93: 264-8.

38. Müller PA, Bröcker EB, Klinker E, Stoevesandt J, Benoit S. Adjuvant treatment of recalcitrant bullous pemphigoid with immunoadsorption. Dermatology 2012; 224: 224-7. http://dx.doi.org/10.1159/000339071

39. Schulz S, Deuster D, Schmidt E, Bonsmann G, Beissert S. Therapeutic effect of etanercept in anti-laminin 5 (laminin 332) mucous membrane pemphigoid. Int J Dermatol 2011; 50: 112931. http://dx.doi.org/10.1111/j.1365-4632.2010.04707.x

40. Vojáèková N, Fialová J, Vaòousová D, Hercogová J. Pemphigus vulgaris treated with adalimumab: case study. Dermatol Ther 2012; 25: 95-7. http://dx.doi.org/10.1111/j.1529-8019.2012.01433.x

41. Fania L, Zampetti A, Guerriero G, Feliciani C. Alteration of cholinergic system in keratinocytes cells produces acantholysis: a possible use of cholinergic drugs in pemphigus vulgaris. Antiinflamm Antiallergy Agents Med Chem 2012; 11: 238-42. http://dx.doi.org/10.2174/1871523011202030238

42. Bech R, Nielsen JB, Peterslund NA, Steiniche T, Deleuran M, d'Amore F. Alemtuzumab is effective against severe chronic lymphocytic leukaemia-associated paraneoplastic pemphigus. Br J Dermatol 2013; 169: 469-72. http://dx.doi.org/10.1111/bjd.12324

43. Hashimoto T, Kawakami T, Koga H, Ohyama B, Hamada T, Dainichi T, et al. Therapeutic effect of mizoribine on pemphigus vulgaris and pemphigus foliaceus. Dermatol Ther 2012; 25: 3825 . 